\title{
3D Visualization of Neurites in Mouse Primary Hippocampal Neuron Cultures Using Cryo-Electron Tomography
}

\author{
Yue Yu ${ }^{1}$, Barkha Yadav-Samudrala ${ }^{2}$, Rick Meeker ${ }^{2}$, Sylvia Fitting ${ }^{2}$ and Lena Kourkoutis ${ }^{1}$ \\ ${ }^{1}$ Cornell University, Ithaca, New York, United States, ${ }^{2}$ University of North Carolina at Chapel Hill, \\ Chapel Hall, North Carolina, United States
}

Each neuron in the human brain may connect to thousands of other neurons making up a complex neuronal network through which signals are passed. Understanding the microstructure of functional units of this network will provide important information about signal transmission, communication and development. Cryo-electron tomography (cryo-ET) has been demonstrated as a powerful tool to probe and visualize the three-dimensional (3D) architecture and organization of sub-cellular components within their near native environment. Recent work has used cryo-ET to study the ultrastructure of neurites and synapses in mature rat hippocampal neuron cultures [1,2]. Here, we present a cryo-ET study of neurites in primary mouse cultures and focus on the early stages of development at 8 days in vitro (DIV), during the neuron differentiation period and prior to the formation of most synaptic structures. While neurons harvested from rats are more stable in culture, we use mice to leverage the rapid development of transgenic and knockout technology which provides new avenues to explore fundamental aspects of neuronal network function.

3D imaging by cryo-ET was performed on dissociated primary mouse hippocampal neurons grown directly on TEM-grids coated with poly-L-lysine. Figure 1A shows an optical phase image of a specimen prior to plunge-freezing, demonstrating typical cell density and cell health conditions in our cultures. The cell density is carefully optimized to obtain a proper specimen thickness after vitrification, balancing the complexities of the neuronal network and the accessibilities for cryo-ET. Fluorescence microscopy was used to confirm that almost all of the cells grown on TEM grids are neurons. The presence of neurons was assessed by microtubule associated protein 2 immunostaining (MAP2ab, green) and a small fraction of astroglial cells was identified by glial fibrillary acidic protein staining (GFAP, red; Fig. 1B).

All of the cryo-ET imaging in this work was performed on a FEI Talos Arctica operating at $200 \mathrm{kV}$ with a Gatan K3 direct electron detector. Tilt-series were recorded using SerialEM [3] from $69^{\circ}$ to $-60^{\circ}$ with $2^{\circ}$ increments and a total electron dose of $\sim 80 \mathrm{e} / \AA^{2}$. To improve tracking accuracy and avoid problems with reaching high tilts, we make use of the bi-directional strategy, running tilt-series from zero-tilt in both directions. To better preserve neurons in their native states in the on-grid cell culture, we did not apply gold nanoparticles as tilt-series fiducial markers. The alignment of tilt-series is instead accomplished with fiducial-less patch alignment in IMOD [4]. Reconstructed tomographic slices of a neurite are presented in Fig. 2A-C. The neurite is found at the end of a thin protrusion branching out from the network (Fig. 2D). Compared to a projection image shown in Fig. 2E, the tomogram reveals the internal cell architecture and organelles such as vesicles, vacuoles, microtubules and mitochondria are identified. In the tomogram, actin filaments are also observed, growing along the cell membrane to support the cell structure. Actin filaments are found to branch out from a bundle as the cell encounters a hole in the carbon film of the TEM grid. Microtubules, clearly identified in the tomographic reconstruction, run mostly in the same direction with the protrusion, consistent with one of their major roles in cellular transport.

This work presents our initial steps to probe the ultrastructure of neuronal networks in the early stages of development. For full access to more complex and mature networks, sample thinning by cryo-focused ion beam milling (cryo-FIB) will be required [5]. [6] 

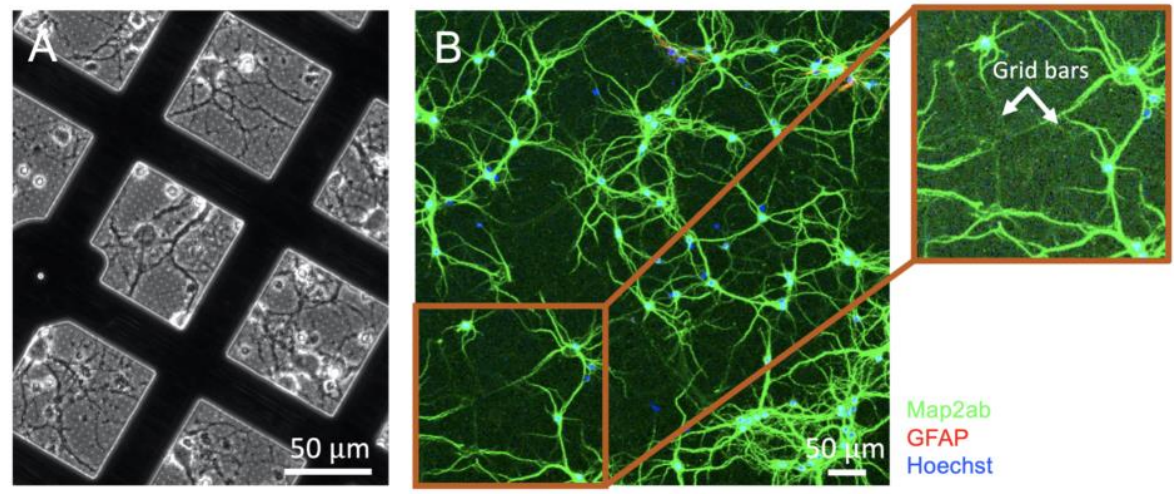

Figure 1. Phase (A) and fluorescence images (B) of dissociated primary hippocampal neurons grown on coated TEM grids. In the fluorescence image (B), the green signal identifies neurons, the red astroglial cells and the blue cell nuclei. TEM grid bars are identifiable (B, right), however, the effect of the TEM grid on the growth of neurons appears minor.
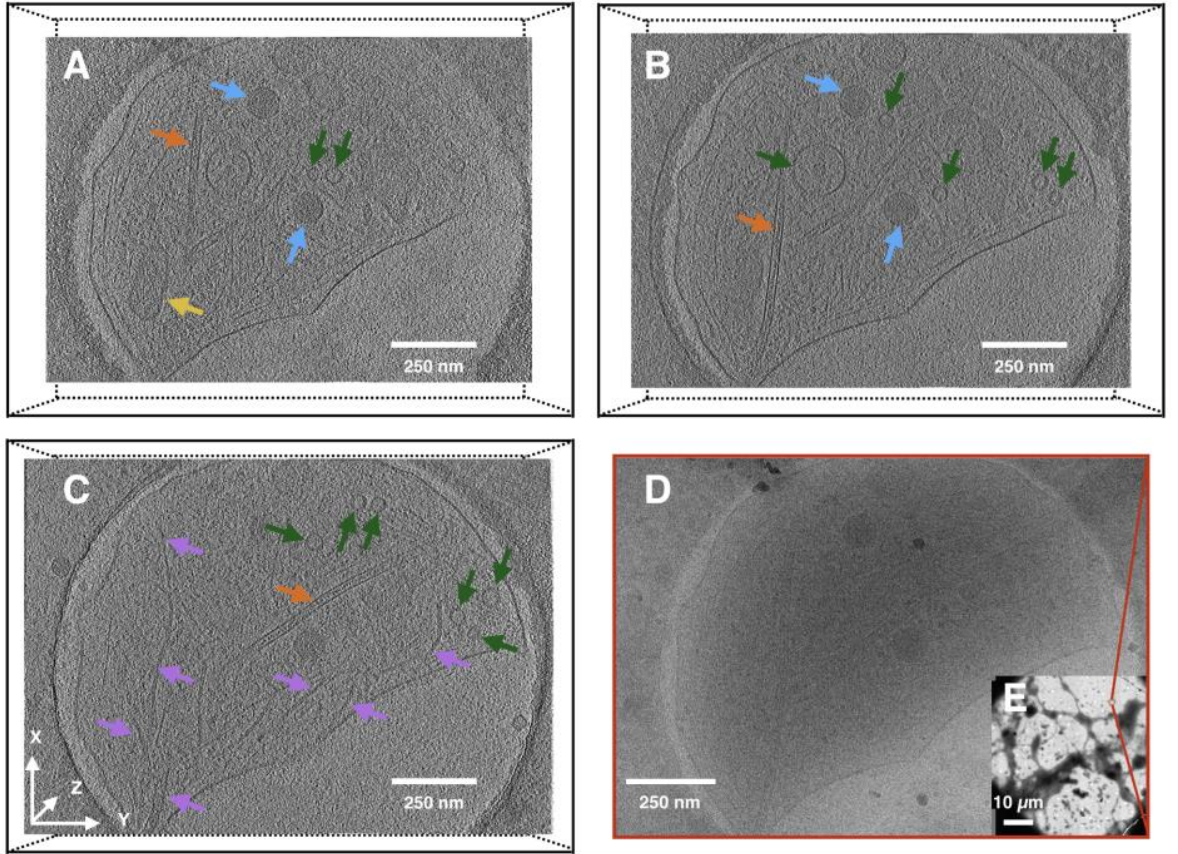

$\uparrow$ Vacuole $\uparrow$ Vesicle $\uparrow$ Microtubules $\uparrow$ Filaments $\uparrow$ Mitochondria

Figure 2. (A-C) Tomographic slices of the neurite reveal the organization of vesicles, vacuoles, mitochondria, actin bundles and microtubules inside the cell. (D) $0^{\circ}$-tilt projection image within the tiltseries. (E) Low-magnification cryo-TEM image shows the neurite of interest is located at the end of a thin protrusion.

\section{References}

[1] S. H. Shahomradian, et al., Journal of Visualized Experiments 84 (2014), e50783.

[2] C. Tao, et al., the Journal of Neuroscience, 38 (2018), p. 1493-1510.

[3] D.N. Mastronarde, Journal of Structural Biology 152 (2005), p. 36-51.

[4] J. R. Kremer, et al., Journal of Structural Biology 116 (1996), p. 71-76.

[5] J. M. Noble, et al., Molecular Microbiology 109 (2018), p. 812-825.

[6] Work supported through the National Institute on Drug Abuse grant R21 DA045597. 